\title{
Impaired phagocytosis of apoptotic cells causes accumulation of bone marrow-derived macrophages in aged mice
}

\author{
Ok-Hee Kim ${ }^{1}$, Hyojung Kim ${ }^{1}$, Jinku Kang ${ }^{1}$, Dongki Yang ${ }^{2}$, Yu-Hoi Kang ${ }^{3}$, Dae Ho Lee ${ }^{1}$, Gi Jeong Cheon ${ }^{4}$, \\ Sang Chul Park ${ }^{5, *}$ \& Byung-Chul Oh ${ }^{1,2, *}$ \\ ${ }^{1}$ Lee Gil Ya Cancer and Diabetes Institute, College of Medicine, Gachon University, ${ }^{2}$ Department of Physiology, College of Medicine, Gachon \\ University, Incheon 21999, ${ }^{3}$ Samsung Advanced Institute of Technology, Samsung Electronics Co. Ltd., Suwon 16678, ${ }^{4}$ Department of \\ Nuclear Medicine, Seoul National University College of Medicine, Seoul 03080, ${ }^{5}$ Well Aging Research Center, DGIST, Daegu 42988, Korea
}

\begin{abstract}
Accumulation of tissue macrophages is a significant characteristic of disease-associated chronic inflammation, and facilitates the progression of disease pathology. However, the functional roles of these bone marrow-derived macrophages (BMDMs) in aging are unclear. Here, we identified agedependent macrophage accumulation in the bone marrow, showing that aging significantly increases the number of M1 macrophages and impairs polarization of BMDMs. We found that age-related dysregulation of BMDMs is associated with abnormal overexpression of the anti-inflammatory interleukin-10. BMDM dysregulation in aging impairs the expression levels of pro-inflammatory cytokines and genes involved in B-cell maturation and activation. Phagocytosis of apoptotic Jurkat cells by BMDMs was reduced because of low expression of phagocytic receptor $\mathrm{CD14}$, indicating that increased apoptotic cells may result from defective phagocytosis of apoptotic cells in the BM of aged mice. Therefore, CD14 may represent a promising target for preventing BMDM dysregulation, and macrophage accumulation may provide diagnostic and therapeutic clues. [BMB Reports 2017; 50(1): 43-48]
\end{abstract}

\section{INTRODUCTION}

Macrophages protect the host from foreign pathogens, phagocytose apoptotic cells and cellular debris, and promote macrophage proliferation in response to environmental cues (1). Macrophages are highly diverse and heterogeneous cells that promptly change their function depending on their

*Corresponding authors. Byung-Chul Oh, Tel: +82-32-899-6074; Fax: +82-32-899-6075; E-mail: bcoh@gachon.ac.kr, Sang Chul Park, Tel: +82-32-899-6074; Fax: +82-32-899-6075; E-mail: scpark@snu. ac.kr

https://doi.org/10.5483/BMBRep.2017.50.1.167

Received 29 September 2016, Revised 21 October 2016, Accepted 14 November 2016

Keywords: Aging, CD14, IL-10, Macrophages, Phagocytosis location in the body (2). Depending on the stimulus, they are categorized into two phenotypes: classically activated macrophages (M1) and alternatively activated macrophages (M2) (3). M1/M2 classification is based on the cytokines associated with macrophage phenotypes (3). Bacterial lipopolysaccharide (LPS) and/or interferon- $\gamma$ stimulate production of M1 macrophages, which promote pro-inflammatory cytokines and reactive oxygen species (ROS) with antimicrobial activity. Immunosuppressive cytokines such as interleukin (IL)-4 and IL-13 yield M2 macrophages, which are mostly associated with tissue repair and suppressing inflammation (4). M2 macrophages show high phagocytic activity but produce low levels of pro-inflammatory cytokines and high levels of the anti-inflammatory cytokine IL-10 (5). Macrophage phenotype is regulated by a combination of local tissue signals and disease programming of resident macrophages, resulting in characteristic tissue-dependent macrophage phenotypic alterations (6). Therefore, macrophages are highly responsive to local tissue signals and alter their phenotypes accordingly.

Macrophages play essential roles in the rapid and efficient removal of apoptotic cells without causing damage to local tissues or initiating immune responses. Therefore, impairment of the phagocytic clearance of apoptotic cells may lead to the development of a variety of diseases, such as chronic inflammation, autoimmunity, and developmental abnormalities (7). When the number of apoptotic or dying cells increases beyond the normal rate, cellular debris is cleared by resident macrophages or those recruited from the circulation or local proliferation. Tissue macrophages are capable of self-renewal and proliferate locally under various disease conditions, such as parasitic infection, obesity, atherosclerosis, and cancer (8-10). These macrophages exhibit the phenotype of alternatively activated M2 macrophages, a process driven by Th2 cytokines such as IL-4 and IL-10.

The contribution of macrophages to disease progression raises an important question about the nature of macrophage accumulation during aging. This study investigated the functional role of bone marrow (BM)-derived macrophages (BMDMs) in age-associated changes. We monitored the expression levels of various cytokines and surface receptors

ISSN: 1976-670X (electronic edition)

Copyright (c) 2017 by the The Korean Society for Biochemistry and Molecular Biology

(c) This is an open-access article distributed under the terms of the Creative Commons Attribution Non-Commercial License (http://creativecommons.org/licenses/by-nc/4.0) which permits unrestricted non-commercial use, distribution, and reproduction in any medium, provided the original work is properly cited. 
that affect macrophage phenotypes and the phagocytic function of BMDMs toward apoptotic Jurkat cells during aging. Using the macrophage-specific tracer, calcium-iron inositol hexakisphosphate $\left(\mathrm{IC}^{\mathrm{I}} \mathrm{IP}_{6}\right)(11)$, we showed an age-dependent increase in BMDMs with impaired M1/M2 polarization and decreased phagocytosis of apoptotic cells in aged BM. These results might provide novel insights into the diverse physiological and pathological roles of BMDMs with potential therapeutic and diagnostic benefits in aging.

\section{RESULTS}

\section{BMDM accumulation and dysregulation of macrophage polarization in aged mice}

Macrophages undergo significant functional changes during aging; however, age-related macrophage distribution and histopathology in BM microenvironments remain unclear. As a macrophage-specific tracer (Fig. 1A), intravenously injected IC-IP ${ }_{6}$ demonstrated maximal IC-IP ${ }_{6}$ uptake at $4 \mathrm{~h}$ in the liver of young mice (Supplementary Fig. 1). Other tissues, including the brain, heart, lung, kidney, lymph node, and thymus, showed very low levels of IC-IP 6 uptake (Supplementary Fig. 1), as verified by Prussian blue staining to detect the presence of iron in IC-IP $\mathrm{P}_{6}$. IC-IP $\mathrm{P}_{6}$ was injected intravenously into young and aged mice. IC-IP 6 was engulfed by tissue macrophages, especially hepatic Kupffer cells, in a manner that did not differ significantly during aging (Fig. 1B). In contrast, the BM of aged mice had a markedly increased number of Prussian bluepositive cells compared with young mice (Fig. 1C, 1D). IHC confirmed the age-dependent increase in BMDMs in the BM (Fig. 1E). These results strongly suggest that aging significantly increases the number of BMDMs. We investigated the effects of aging on macrophage distribution in BM by analyzing the percentage of BMDMs and the frequency of M1 and M2 macrophages in young and aged mice. We found a significant increase in the percentage of $\mathrm{CD} 11 \mathrm{~b}^{+} \mathrm{MHCll}^{+} \mathrm{F} 4 / 80^{+}$cells in the $\mathrm{BM}$ of aged mice compared with that in young mice (Fig. 1F). The analysis of BMDM M1 and M2 phenotypes showed that aging had a significant impact on the polarization of BMDMs (Fig. 1G). The percentage of M1 macrophages was approximately 2.5-fold higher in the BM of aged mice than that in young mice, whereas no significant increase in $M 2$ macrophages was found in aged mice (Fig. 1G). These results strongly suggest that the age-related impairment of M1/M2 BMDM polarization may dysregulate host immune responses and the microenvironment.

\section{Dysregulation of cytokines and surface receptors in BMDMs in aged mice}

Aging is associated with increased levels of circulating inflammatory cytokines including IL-6 and TNF- $\alpha$ (12); thus,


d
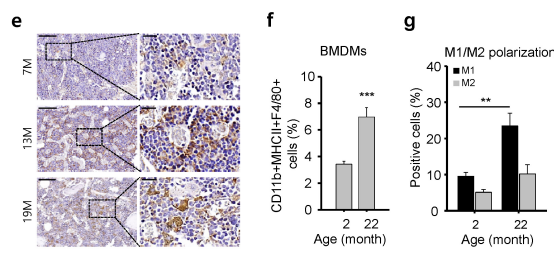

Fig. 1. Increased numbers and dysregulation of bone marrow (BM)-derived macrophages (BMDMs) in aged mice. (A) Schematic illustration of $I C-I P_{6}$. (B) IC-InsP $P_{6}$ accumulation in the liver was visualized by double-staining with Prussian blue (iron) and the macrophage-specific anti-F4/80 antibody (brown). Scale bars: top, $50 \mu \mathrm{m}$; bottom, $20 \mu \mathrm{m}$. (C) Prussian blue staining for assessment of IC-IP 6 uptake by BMDMs in decalcified femur sections from age-matched mice. (D) Quantification of Prussian blue-positive cells per $\mathrm{mm}^{2}$ in the BM of mice intravenously injected with 10 $\mu \mathrm{M} I \mathrm{C}-\mathrm{IP}_{6}$, mean $\pm \mathrm{SE}\left(\mathrm{n}=10, * * * \mathrm{P}<10^{-3}\right)$. (E) Immunohistochemical staining of $\mathrm{F} 4 / 80$ in decalcified femur sections from age-matched mice. Scale bars: top, $200 \mu \mathrm{m}$; bottom, $50 \mu \mathrm{m}$. (F) Frequency of total BMDMs assessed by flow cytometric analysis of $\mathrm{CD}_{11 \mathrm{~b}^{+} \mathrm{MHClI}}{ }^{+} \mathrm{F} 4 / 80^{+}$cells in the $\mathrm{BM}$ of age-matched mice, mean $\pm \mathrm{SE}\left(\mathrm{n}=6-7,{ }^{* * * P}<10^{-3}\right)$. (G) M1 and M2 macrophage polarization assessed by flow cytometric analysis of $\mathrm{CD}^{\prime} 1 \mathrm{~b}^{+}$/ $\mathrm{CD}^{2} 6^{+}$and $\mathrm{CD} 11 \mathrm{~b}^{+} / \mathrm{CD}^{2} 26^{-}$macrophages from $\mathrm{MHCll}^{+} \mathrm{F} 4 / 80^{+}$ cells in BM cells of age-matched mice, respectively, mean $\pm \mathrm{SE}$ ( $\mathrm{n}=6-7, * * \mathrm{P}<0.01)$.
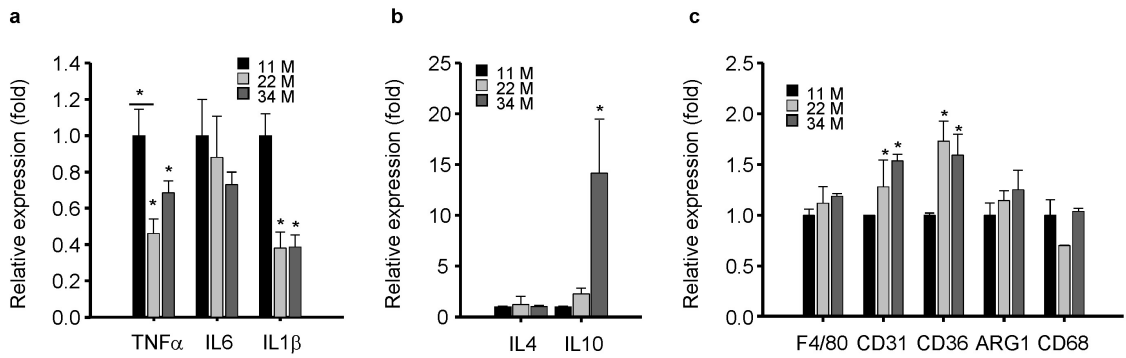

Fig. 2. Dysregulated mRNA expression of cytokines and surface receptors in aged mice. (A-C) The mRNA levels of pro-inflammatory cytokines (A), antiinflammatory cytokines (B), and surface receptors $(C)$ in the $B M$ of age-matched mice were measured by qPCR. All mRNA levels were normalized to cyclophilin A, mean \pm SE $(n=4, * P<$ 0.05). 
we assessed the expression levels of cytokines and chemokines during aging. The expression levels of pro-inflammatory cytokines such as TNF $\alpha$, IL-6, and IL1 $\beta$ were reduced dramatically in BMDMs of aged mice compared with young mice (Fig. 2A), indicating that the dysregulation of BMDMs impairs the expression of pro-inflammatory cytokines. The expression profiles of anti-inflammatory cytokines IL-4 and IL-10 were also dysregulated in the BM of aged mice. IL-4 expression was markedly reduced in aged compared with young mice, but IL-10 expression increased significantly with age (Fig. 2B). As IL-10 inhibits the synthesis of pro-inflammatory cytokines (13), age-related dysregulation in BMDMs may be closely associated with the abnormal overexpression of anti-inflammatory IL-10 (Fig. 2B). Macrophages respond effectively to immune stimuli via surface receptors (3). We found that macrophage markers CD31 and CD36 increased significantly with aging, F4/80 and ARG1 increased slightly, and CD68 showed no significant change with aging (Fig. 2C).

\section{Upregulation of genes associated with B-cell activation, survival, and surface antigen production}

Surface antigens on macrophages play important roles in antigen presentation and activation of the adaptive immune response $(1,2)$. Surface antigens CD19, CD22, IL7R, CD72, CD79A, and CD79B were overexpressed in an age-dependent manner in the BM (Fig. 3A). Furthermore, the expression levels of B-cell activating factor (BAFF) and BAFF receptor were
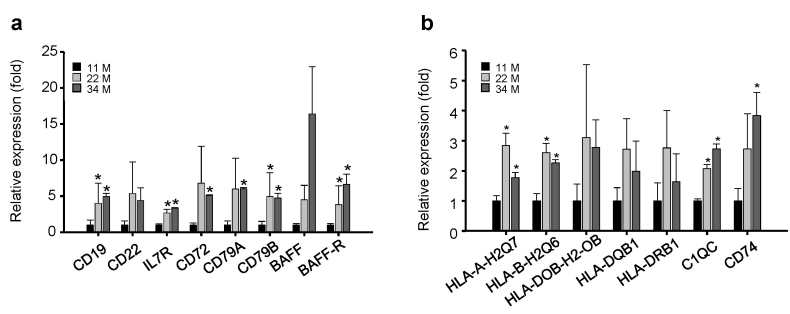

c

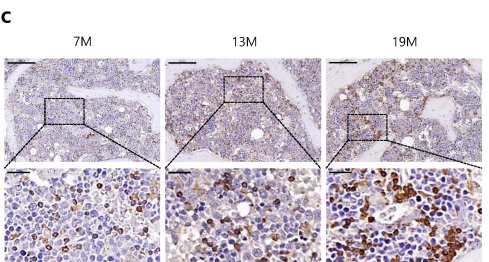

d

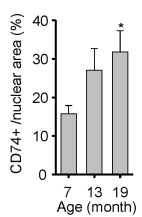

Fig. 3. mRNA expression of genes associated with B-cell activation, survival, and antigen production is upregulated in the $\mathrm{BM}$ of aged mice. (A) The mRNA levels of genes involved in B-cell development and function (A), B-cell activating factor (BAFF), and BAFF receptor (BAFF-R) (B), and MHC class II molecules. (C) Immunohistochemical staining of CD74 in decalcified femur sections from age-matched mice. Scale bars: top, $200 \mu \mathrm{m}$; bottom, $50 \mu \mathrm{m}$. (D) Quantification of $\mathrm{CD}_{4}^{+}$cells per $\mathrm{mm}^{2}$ in decalcified femur sections from age-matched mice, mean \pm SE ( $n$ $=10, * \mathrm{P}<0.05)$ increased in an age-dependent manner. In addition, the expression levels of BAFF and BAFF-R were increased significantly in the BM of aged mice (Fig. 3A). BAFF is responsible for B-cell survival, maturation, and antibody production (14); therefore, excessive expression of BAFF might induce abnormally high antibody production, which could lead to autoimmune diseases especially those associated with aging (3). Furthermore, MHC class II molecules such as HLA-A-H2Q7, HLA-B-H2Q6, C1QC, and CD74 were increased significantly in the BM of aged mice (Fig. 3B), showing that genes involved in B-cell maturation and antigen production are highly upregulated in the BM of aged mice. CD74 plays a role in antigen presentation (15), and its loss inhibits B-cell development (16). In our study, IHC staining revealed a significant increase in CD74 expression in the BM of aged mice compared with young mice (Fig. 3C, D), suggesting that CD74 could be a potential therapeutic target in the BM for activation of B-cell survival and maturation during aging.

\section{BMDM accumulation and phagocytic defects in apoptotic cells in aged mice}

We hypothesized that both BMDM accumulation and increased expression of anti-inflammatory cytokines in aged mice are involved in the clearance of apoptotic cells or cellular debris. Using a fluorescently labeled suicide inhibitor with a DEVD sequence, the specific cleavage site for caspases 3 and 7 (FAM-FLICA, ImmunoChemistry Tech), we found that apoptosis was increased significantly in the $\mathrm{BM}$ of aged mice (Fig. 4A). Furthermore, immunohistochemical staining of cleaved caspase 3 showed an increased rate of apoptosis in the BM of aged compared with control mice (Fig. 4B, C). Consistent with previous reports suggesting that aging increases the rate of apoptosis $(7,17)$, these results implicate that BMDM accumulation in the BM of aged mice might be due to the high incidence of apoptosis and low clearance of BMDMs. The rapid and efficient phagocytic clearance of cell debris is an essential process for maintaining tissue health and preventing autoantigen production (18). Therefore, we assessed the phagocytic function of BMDMs toward apoptotic Jurkat cells after inducing apoptosis using camptothecin. Engulfed and non-engulfed apoptotic Jurkat cells were distinguished by labeling apoptotic Jurkat cells with a $\mathrm{pH}$-sensitive dye (pHrodo) that emits red fluorescence, only when the engulfed cells are localized inside the phagosome of macrophages $(\mathrm{pH}$ 5) (19). After inducing apoptosis, the BMDMs from young mice efficiently engulfed most of the apoptotic Jurkat cells (Fig. 4D). The BMDMs from aged mice displayed dramatically decreased engulfing activity (Fig. 4E), indicating that this phagocytic function decreases with aging and leads to increased numbers of apoptotic cells in the BM of aged mice. To address the molecular mechanism underlying the impaired phagocytosis of apoptotic cells, we analyzed the expression levels of the genes associated with phagocytic clearance of apoptotic cells (20). The expression of TIMD4 and Stabilin-2 

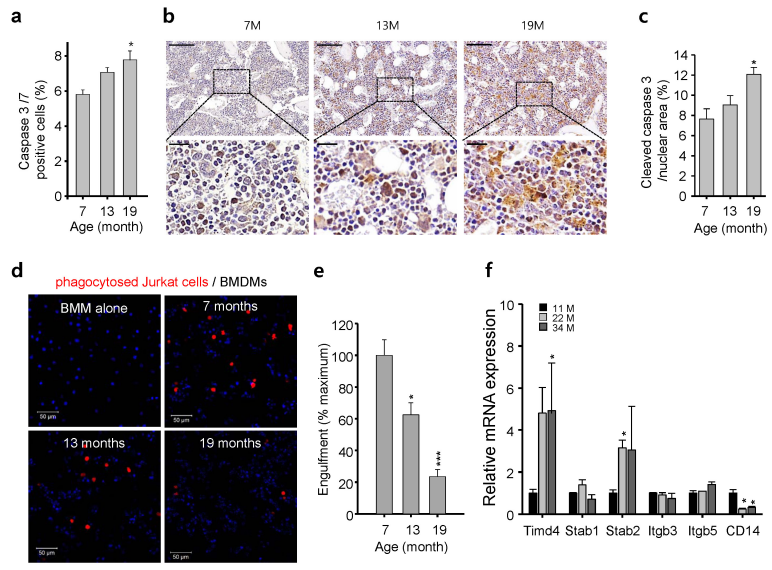

Fig. 4. Age-dependent accumulation of BMDMs may result from phagocytic impairment of apoptotic cells through reduced levels of the phagocytic receptor CD14. (A) Rate of apoptosis assessed using a fluorescently labeled suicide inhibitor with a DEVD sequence, the specific cleavage site for caspases 3 and 7 , in BM cells of age-matched mice, mean \pm SE $(n=3-4, * P<0.05)$. (B) Immunohistochemical staining of cleaved caspase 3 in decalcified femur sections from age-matched mice. Scale bars: top, $200 \mu \mathrm{m}$; bottom, $50 \mu \mathrm{m}$. (C) Quantification of the area (\%) of cleaved caspase 3 staining in decalcified femur sections from age-matched mice, mean $\pm \mathrm{SE}(\mathrm{n}=10, * \mathrm{P}<0.05)$. (D) Representative images of pHrodo-labeled apoptotic Jurkat cell phagocytosed (red) by BMDMs in age-matched mice. Scale bar: $50 \mu \mathrm{m}$. (E) Quantification of phagocytosis, mean \pm SE $(n=6-8$, $* * * \mathrm{P}<10^{-3}$ ). (F) mRNA levels of genes associated with phagocytic clearance of apoptotic cells in BMs of age-matched mice measured by $q P C R$, mean \pm SE $(n=4, * P<0.05)$.

were dramatically upregulated on macrophages, whereas the expression levels of BAI-1, BAI-3 and TIMD1 were not detected (data now shown), suggesting these genes are not expressed in BMDMs. Most importantly, the expression of CD14, the receptor highly expressed on macrophage (21), was dramatically decreased in the BM of aged mice (Fig. 4F), confirming an age-related decline in phagocytosis receptors (22). Therefore, the impaired phagocytic function of BMDM toward apoptotic cells might involve low CD14 expression in aged mice.

\section{DISCUSSION}

Tissue macrophage accumulation is a characteristic of diseaseassociated chronic inflammation including atherosclerosis, cancer, chronic kidney disease, obesity, and type 2 diabetes. Macrophage accumulation is considered a consequence of both monocyte migration and local proliferation, which can induce disease pathology. Therefore, monitoring tissue macrophages in aged animals might reveal the underlying mechanisms of tissue aging and age-related pathologies.

This study found that aging causes significant increases in BMDM numbers and impaired M1/M2 polarization in BM.
Dysregulated M1/M2 polarization alters the secretory pattern of pro- and anti-inflammatory cytokines. Notably, overexpression of IL-10 in aged mice may suppress the expression of pro-inflammatory cytokines by the increased number of $\mathrm{M} 1$ macrophages $(4,13,22,23)$. These findings suggest that the abnormal overexpression of anti-inflammatory IL-10 in the BM of aged mice could be a cause of dysregulated polarization of BMDMs.

Macrophages play important roles in host defenses against invading pathogens, clearing cellular debris, and antigen presentation to induce the adaptive immune response $(1,24)$. We observed age-dependent overexpression of surface antigens associated with B-cell survival, maturation, and autoantibody production in the BM of aged mice. Concomitantly, MHC class II surface antigen (HLAs, C1Q, and CD74) expression levels were increased in the $B M$, implying that dysregulation of BMDMs in aged mice plays an important role in B-cell activation and autoantigen presentation. Experimental evidence showed that age-related dysregulation of BMDMs might play a critical role in age-related impairment of adaptive immunity and progression of age-related diseases $(13,14,23)$.

The significantly decreased phagocytosis of apoptotic Jurkat cells by BMDMs from aged mice suggests that the defective clearance of apoptotic cells leads to increased apoptotic cell numbers in the BM of aged mice (23). However, a previous study reported no impairment of BMDM-mediated phagocytosis of fluorescent particles in aged mice (22). We suggest that this conflicting data could be related to the different phagocytosis assay methods employed. As anti-inflammatory IL-10 dysregulates the phagocytic function of peritoneal macrophages (22, $25)$, high expression of IL-10 in the BM of aged mice may aggravate the defective phagocytosis of apoptotic Jurkat cells by BMDMs. Although IL-10 increases expression of the phagocytic receptor CD14 and stimulates phagocytic function toward apoptotic cells (26), there are several conflicting results regarding IL-10. Decreased subsets of $\mathrm{CD} 14^{++} \mathrm{CD} 16^{-}$ monocytes were found in patients with heart failure despite high levels of IL-10 (26), and increased expression of IL-10 was detected in CD14 knockout mice (27). Therefore, our findings suggest that dysregulation of IL-10 in aged tissues leads to weak expression of the phagocytic receptor CD14. Low levels of CD14 in aged BMDMs may underlie the age-associated impairment of apoptotic cell phagocytosis (23).

This study demonstrated that aging causes increased BMDM numbers, dysregulated BMDM polarization, increased antiinflammatory IL-10 expression, impaired phagocytosis of apoptotic cells, and reduced expression of CD14. These findings might provide new molecular targets with potential therapeutic and diagnostic benefits for age-associated diseases.

\section{MATERIALS AND METHODS}

Antibodies and reagents

The following antibodies were used for Western blotting, 
confocal microscopy, and flow cytometry analyses: mouse F4/80 (BM8, eBioscience, San Diego, CA, USA), $\beta$-actin (AC-15, Sigma-Aldrich), mouse CD74 (05-591, Millipore), human Annexin V (ab14196, Abcam), and cleaved caspase-3 (D175, Cell Signaling). For chemical induction of apoptosis, cells were treated with $10 \mu \mathrm{M}$ camptothecin at $37^{\circ} \mathrm{C}$ for $16 \mathrm{~h}$ using an apoptosis inducer kit (ab102480; Abcam).

\section{Animal care and use}

WT C57BI/6 mice were from Orient (Korea). Male mice were studied under protocols approved by the animal ethics committee of Gachon University, Lee Gil Ya Cancer and Diabetes Institute. We used mice aged 2-12 months as the young control group and defined aged mice as old as 18-34 months.

\section{Preparation of IC-IP 6}

Based on our isothermal titration calorimetry results (28), a 10 $\mathrm{mM}$ solution of $\mathrm{Fe}^{3+}-\mathrm{IP}_{6}$ was prepared by mixing equimolar concentrations of $\mathrm{Fe}^{3+}$ and phytate $(\mathrm{pH}$ 6.0). To minimize chelation in the blood, an equimolar concentration of $\mathrm{Ca}^{2+}$ was added to the $\mathrm{Fe}^{3+}-\mathrm{IP}_{6}$ solution and adjusted to $\mathrm{pH} 6.0$ by adding $1 \mathrm{~N} \mathrm{NaOH}$.

\section{Assessment of IC-IP $\mathbf{6}_{\mathbf{6}}$ uptake by $\mathrm{BMDMs}$}

IC-IP 6 (10 $\left.\mathrm{mg} \cdot \mathrm{kg}^{-1}\right)$ was administered intravenously to 6-, 12-, and 18-month-old C57BL/6 mice. Femurs were harvested after $4 \mathrm{~h}$, fixed in 10\% neutral-buffered formalin for $48 \mathrm{~h}$, decalcified in $10 \%$ EDTA ( $\mathrm{pH} 7.0$ ) for $4-5$ weeks at $4^{\circ} \mathrm{C}$, and embedded in paraffin for immunohistochemistry (IHC) (28). IC-IP ${ }_{6}$ in $\mathrm{BM}$ sections was detected by Prussian blue staining.

\section{Generation of apoptotic cells}

Jurkat T cells were cultured in RPMI media supplemented with $10 \%$ FBS and $1 \%$ penicillin/streptomycin. Apoptosis was induced with camptothecin $(10 \mu \mathrm{M})$ at $37^{\circ} \mathrm{C}$ for $16 \mathrm{~h}$.

\section{Detection of apoptotic cells in BM tissues}

For IHC, antigen retrieval was performed in Tris/EDTA $(\mathrm{pH}$ 9.0) with 2-3 min of boiling. Bone sections (2.5- $\mu \mathrm{m}$ thick) from 6-, 12-, and 18-month-old mice were treated with serum-free protein blocking solution (Dako), incubated with anti-cleaved caspase 3 rabbit antibodies (9661, 1:200; Millipore) followed by horseradish peroxidase-conjugated secondary antibodies for $30 \mathrm{~min}$, and detected with 3,3'-diaminobenzidine tetrahydrochloride (Dako).

\section{In vitro phagocytosis assay}

Jurkat T cells were cultured overnight at a density of $5 \times$ $10^{6} / \mathrm{ml}$ and treated with camptothecin $(10 \mu \mathrm{M})$ for $16 \mathrm{~h}$. Growing or apoptotic Jurkat T cells were washed with PBS and incubated with $5 \mu \mathrm{M} / \mathrm{ml}$ pHrodo Red AM (P353721, Life Technologies) for $30 \mathrm{~min}$ at $37^{\circ} \mathrm{C}$. The pHrodo-labeled Jurkat T cells were washed with live cell imaging solution (A14291DJ,
Life Technologies). BMDMs were cultured on 12-well plates $\left(5 \times 10^{5} /\right.$ well) for $16 \mathrm{~h}$ before treatment with $0.5 \mu \mathrm{g} / \mathrm{ml}$ LPS for $6 \mathrm{~h} \mathrm{(18)}$. For analysis of phagocytosis, pHrodo-labeled Jurkat T cells were co-incubated with BMDMs for 1.5-2 $\mathrm{h}$, washed with cold PBS, fixed in 10\% neutral-buffered formalin for $10 \mathrm{~min}$, counterstained with DAPI to visualize the nuclei, and analyzed by confocal microscopy (Zeiss LSM 710).

\section{Macrophage polarization and apoptosis analysis}

BMDMs were isolated from 2-month-old or 22-month-old $\mathrm{C} 56 / \mathrm{BL}$ mice. Staining was performed at $4^{\circ} \mathrm{C}$ for $30 \mathrm{~min}$. The M1/M2 macrophage phenotype was analyzed using antiCD11b-FITC (M1/70), anti-F4/80-PE-Cy7 (BM8), major histocompatibility complex (MHC) class II, and CD206-APC (C068C2) antibodies. All antibodies were from eBioscience except for anti-CD206 (Biolegend). For apoptotic cell analysis, BM cells were isolated from 7-, 13-, and 19-month-old C57/BL6 mice and co-stained with the caspase 3/7-specific Vybrant-FAM substrate, a fluorescein-conjugated caspase inhibitor, FAM-DEVD-fluoromethyl ketone (V35118, Molecular Probes) and Alexa 647-conjugated Annexin V (29). The cells were analyzed by flow cytometry using the FACS LSRII (BD Bioscience) and Flowjo software.

\section{Quantitative PCR (qPCR) analysis of cytokines and surface receptors}

Total RNA was treated with RQ1 RNase-free DNase (Promega) and reverse transcribed using the PrimeScript First-Strand cDNA Synthesis kit (TaKaRa Bio). qPCR was performed using SYBR Green PCR Master Mix (TaKaRa Bio) with gene-specific primers (Supplementary Table 1) on the ABI PRISM 7900 Sequence detection system. All samples were analyzed in triplicate, and the results are expressed as means \pm SE.

\section{Statistical analysis}

The error bars on the graphs represent the means \pm SE of at least three independent experiments. Student's $t$-tests were used to determine statistical significance between groups, with $\mathrm{P}<0.05$ set as the cut-off value.

\section{ACKNOWLEDGEMENTS}

This work was supported by the Well Aging Research Center, Samsung Advanced Institute of Technology, the DGIST R\&D program of the Ministry of Science, ICT and Technology of Korea (20160172), and the Korean Health Technology R\&D Project through the Korean Health Industry Development Institute of the Ministry of Health \& Welfare, Korea (HI14C1135).

\section{CONFLICTS OF INTEREST}

The authors have no conflicting financial interests. 


\section{REFERENCES}

1. Wynn TA, Chawla A and Pollard JW (2013) Macrophage biology in development, homeostasis and disease. Nature 496, 445-455

2. Pollard JW (2009) Trophic macrophages in development and disease. Nat Rev Immunol 9, 259-270

3. Gordon S (2003) Alternative activation of macrophages. Nat Rev Immunol 3, 23-35

4. Mosser DM and Edwards JP (2008) Exploring the full spectrum of macrophage activation. Nat Rev Immunol 8, 958-969

5. Mosser DM and Zhang X (2008) Interleukin-10: new perspectives on an old cytokine. Immunol Rev 226, 205-218

6. Jenkins SJ, Ruckerl D, Cook PC et al (2011) Local macrophage proliferation, rather than recruitment from the blood, is a signature of $\mathrm{TH} 2$ inflammation. Science 332, 1284-1288

7. Poon IK, Lucas CD, Rossi AG and Ravichandran KS (2014) Apoptotic cell clearance: basic biology and therapeutic potential. Nat Rev Immunol 14, 166-180

8. Amano SU, Cohen JL, Vangala P et al (2014) Local proliferation of macrophages contributes to obesityassociated adipose tissue inflammation. Cell Metab 19, $162-171$

9. Kim DI, Kim E, Kim YA, Cho SW, Lim JA and Park YJ (2016) Macrophage Densities Correlated with CXC Chemokine Receptor 4 Expression and Related with Poor Survival in Anaplastic Thyroid Cancer. Endocrinol Metab (Seoul) 31, 469-475

10. Mielgo A and Schmid MC (2013) Impact of tumour associated macrophages in pancreatic cancer. BMB Rep 46, 131-138

11. Oh BC, Kim HJ (2010) Magnetic resonance imaging contrast agent with paramagnetic-inositol phosphates complexes. US 12/656,075

12. Renshaw M, Rockwell J, Engleman C, Gewirtz A, Katz J and Sambhara S (2002) Cutting edge: impaired Toll-like receptor expression and function in aging. J Immunol 169, 4697-4701

13. Moore KW, de Waal Malefyt R, Coffman RL and O'Garra A (2001) Interleukin-10 and the interleukin-10 receptor. Annu Rev Immunol 19, 683-765

14. Thangarajh $M$, Gomes A, Masterman T, Hillert J and Hjelmstrom P (2004) Expression of B-cell-activating factor of the TNF family (BAFF) and its receptors in multiple sclerosis. J Neuroimmunol 152, 183-190

15. Zimmermann VS, Rovere P, Trucy J et al (1999) Engagement of $\mathrm{B}$ cell receptor regulates the invariant chain-dependent MHC class II presentation pathway. J
Immunol 162, 2495-2502

16. Shachar I and Flavell RA (1996) Requirement for invariant chain in B cell maturation and function. Science 274, 106-108

17. Lauber K, Blumenthal SG, Waibel M and Wesselborg S (2004) Clearance of apoptotic cells: getting rid of the corpses. Mol Cell 14, 277-287

18. Segawa K, Kurata S, Yanagihashi Y, Brummelkamp TR, Matsuda F and Nagata S (2014) Caspase-mediated cleavage of phospholipid flippase for apoptotic phosphatidylserine exposure. Science 344, 1164-1168

19. Yoon KW, Byun S, Kwon E et al (2015) Control of signaling-mediated clearance of apoptotic cells by the tumor suppressor p53. Science 349, 1261669

20. Arandjelovic S and Ravichandran KS (2015) Phagocytosis of apoptotic cells in homeostasis. Nat Immunol 16, 907-917

21. Gregory CD (2000) CD14-dependent clearance of apoptotic cells: relevance to the immune system. Curr Opin Immunol 12, 27-34

22. Linehan E, Dombrowski $Y$, Snoddy R, Fallon PG, Kissenpfennig A and Fitzgerald DC (2014) Aging impairs peritoneal but not bone marrow-derived macrophage phagocytosis. Aging Cell 13, 699-708

23. Plowden J, Renshaw-Hoelscher M, Engleman C, Katz J and Sambhara S (2004) Innate immunity in aging: impact on macrophage function. Aging Cell 3, 161-167

24. Ghesquiere B, Wong BW, Kuchnio A and Carmeliet P (2014) Metabolism of stromal and immune cells in health and disease. Nature 511, 167-176

25. Popi AF, Lopes JD and Mariano M (2004) Interleukin-10 secreted by B-1 cells modulates the phagocytic activity of murine macrophages in vitro. Immunology 113, 348-354

26. Amir O, Spivak I, Lavi I and Rahat MA (2012) Changes in the monocytic subsets CD14(dim)CD16(+) and CD14(++)CD16(-) in chronic systolic heart failure patients. Mediators Inflamm 2012, 616384

27. Sakai K, Hasebe R, Takahashi Y et al (2013) Absence of CD14 delays progression of prion diseases accompanied by increased microglial activation. J Virol 87, 1343313445

28. Kim OH, Kim YO, Shim JH et al (2010) beta-propeller phytase hydrolyzes insoluble $\mathrm{Ca}(2+)$-phytate salts and completely abrogates the ability of phytate to chelate metal ions. Biochemistry 49, 10216-10227

29. Lee YJ, Lee YJ and Lee SH (2015) Resveratrol and clofarabine induces a preferential apoptosis-activating effect on malignant mesothelioma cells by Mcl-1 down-regulation and caspase-3 activation. BMB Rep 48, 166-171 